\title{
The Dynamics of Bubbles in Periodic Vortex Flowss
}

\author{
K.-K. TIO and J.C. LASHERAS
}

Department of AMES, University of California at San Diego, La Jolla, CA 92093-0411, U.S.A.

A.M. GAÑ́N-CALVO

E.T.S. Ingenieros Industriales, Universidad de Sevilla, 41012 Sevilla, SPAIN

and

A. LIÑÁN

E.T.S. Ingenieros Aeronauticos, Universidad Politécnica de Madrid, 28040 Madrid, SPAIN

\begin{abstract}
To analyze the dynamics of small, spherical, rigid bubbles in a certain class of turbulent shear flows dominated by large scale coherent vortical structures, we model the plane free shear layer with a periodic array of Stuart vortices. The equation of motion of the bubbles is then integrated numerically to obtain the Lagrangian description of the bubbles, the long-term dynamics of which depends on the free-stream Reynolds number, the Stokes number, the gravitational field, and the strength of the vortices. Depending on the values of these four parameters, it is found that either there exists a stable equilibrium point near the center of each vortex, where bubble accumulation occurs, or all bubbles escape from captivity by the vortices. In the limiting case of dominant viscous drag forces, an Eulerian description of the "bubble flow field" is derived. Furthermore, the divergence of this flow field is negative in the neighborhood of a vortex center, where it achieves its minimum. This indicates that bubbles accumulation may indeed exist, and thus qualitatively confirms the more general numerical results obtained without the assumption of dominant viscous drag forces.
\end{abstract}

Key words: bubbles - particles - vortex flows.

\section{Introduction}

In this paper, we examine the dynamics of bubbles in a certain class of turbulent shear flows dominated by large scale vortical structures. To this end, the plane free shear layer between two parallel streams is modeled with a spatially periodic array of vortices given by Stuart's [6] solution to the two-dimensional, steady-state, incompressible Euler equation. We assume that the size of the bubbles and their concentration are sufficiently small so that their mutual interactions and effects on the base flow can be neglected. Furthermore, surface tension is also assumed to be large enough so that the bubbles take the form of a spherical rigid particle.

The motion of heavy particles in a row of Stuart vortices under the influence of gravity has been studied by Gañán-Calvo and Lasheras [2]. It is found that heavy particles can be permanently suspended in motion along open trajectories above the vortices. In the case of bubbles, however, this suspension mode does not exist. Instead, a bubble either escapes due to its buoyancy, or is captured by a vortex and remains trapped at the stable equilibrium point near the center of the vortex. The entrapment of bubbles by a vortex has also been observed in other types of vortical 
flows, such as a Langmuir cellular flow [4] and the flow field generated by a line vortex $[7,8]$.

In this paper, we present the results obtained by numerically integrating the equation of motion of the bubbles. We also investigate the effects of the parameters in the governing equations on the dynamics of the bubbles. These parameters are: the free stream Reynolds number, the Stokes number, the strength of the Stuart vortices, and gravity.

\section{Governing Equations}

In dimensionless form, the dynamics of a small, rigid, spherical bubble is governed by the momentum equation $[1,3]$

$$
\frac{d \mathbf{V}}{d t}=3 \mathbf{u} \cdot \nabla \mathbf{u}+(\mathbf{u}-\mathbf{V}) A f_{\mathbf{d}}+2 B \mathbf{e}_{y}
$$

together with

$$
\frac{d \mathbf{x}_{\mathrm{P}}}{d t}=\mathbf{V}
$$

where $\mathbf{V}=\left(V_{x}, V_{y}\right)$ and $\mathbf{u}=\left(u_{x}, u_{y}\right)$ are the bubble and fluid velocities, respectively, $\mathbf{x}_{\mathbf{P}}$ denotes the instantaneous position of the bubble, and $\mathbf{e}_{y}$ is a unit vector in the $y$-direction. In equation (1), the density of the gas inside the bubble has been ignored in comparison to the fluid density. The non-dimensionalization of equations (1)-(2) is accomplished through the use of the length scale $L=\lambda / 2 \pi$, the time scale $T=L / U_{\infty}$, and the free-stream velocity $U_{\infty}$. Here, $\lambda$ is the distance between the centers of two contiguous vortices. The two dimensionless quantities

$$
A \equiv \frac{36 \nu L}{U_{\infty} d^{2}}, \quad B \equiv \frac{L g}{U_{\infty}^{2}},
$$

where $\nu$ is the fluid viscosity, $d$ the bubble diameter, and $g$ the acceleration of gravity, are the inverse of the Stokes number and the gravitational parameter, respectively. Note that we have chosen gravity to point in the negative $y$ direction. The coefficient $f_{\mathrm{d}}$ accounts for the effect of non-zero Reynolds number on the viscous drag acting on the bubble, and is given by [5]

$$
f_{\mathrm{d}}=\left\{\begin{array}{l}
\frac{2}{3}\left(1+0.1 R e_{\mathrm{r}}\right), \quad R e_{\mathrm{r}}<2.0 \\
0.621 R e_{\mathrm{r}}^{0.22}, \quad 2.0 \leq R e_{\mathrm{r}} \leq 50.0
\end{array}\right.
$$

In equation (4), $R e_{\mathrm{r}}$ is the instantaneous Reynolds number of the bubble based on its velocity relative to the fluid, and is given by $R e_{\mathrm{r}}=|\mathbf{u}-\mathrm{V}| R e$, where $R e \equiv U_{\infty} d / \nu$ is the free stream Reynolds number. 


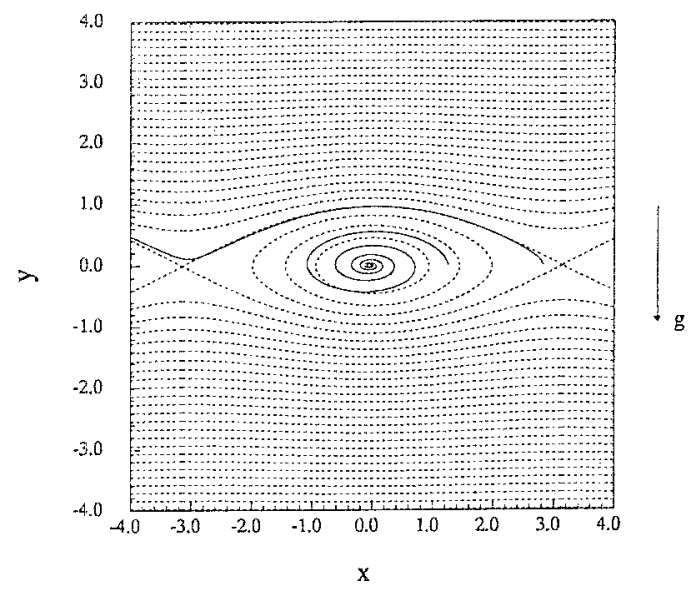

Fig. 1. The trajectories of a bubble trapped by a vortex and one that escapes. The dashed curves are the streamlines. The four parameters are: $k=0.25, A=7, B=0.1, \operatorname{Re}=100$.

The flow field selected for our study consists of a periodic array of Stuart vortices. In dimensionless form, the velocity field is given by

$$
u_{x}=-\frac{\sinh y}{\cosh y-k \cos x}, \quad u_{y}=\frac{k \sin x}{\cosh y-k \cos x},
$$

where the parameter $k$ is a measure of the strength of the vortices. For $k=1$ the flow field consists of a row of point vortices, while $k=0$ corresponds to the $\tanh y$ shear layer.

\section{Results and Discussion}

The dynamical system of (1)-(2) is integrated using the fourth-order Runge-Kutta scheme with a time step of 0.01 . The accuracy of this integration step is examined in a few test cases and appears to be acceptable. To integrate (1)-(2), the initial bubble velocity is chosen to be equal to that of the fluid particle at the initial position of the bubble.

Depending on the values of the four parameters $A, B, k$, and $R e$, either all bubbles escape to $y=\infty$ or some of them are trapped by the vortices. In the entrapment regime, a bubble, depending on its initial position, either escapes to $y=\infty$, or is captured by a vortex and spirals into a fixed point located near the vortex center. These two possibilities are clearly illustrated in Figure 1. We point out that the bubbles trapped by a vortex all asymptotically approach the same stationary point, where they remain at rest. In fact, linear stability analyses of equations (1) and (2) reveal that this stationary point is stable[9].

In Figure 2, we show the regions of entrapment and no entrapment of bubbles in three subsets of the parametric space with fixed $k$ and Re. As an illustration, 


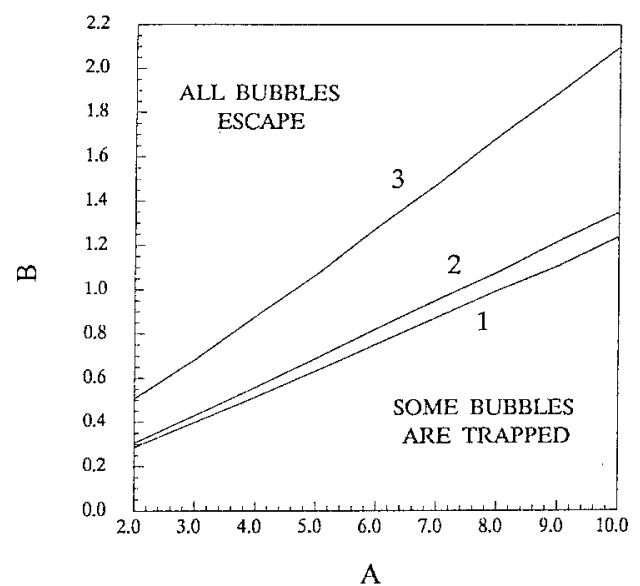

Fig. 2. Regions of entrapment and no entrapment of bubbles in three subsets of the parametric space with fixed $k$ and $R e$. Each curve represents the boundary between the two regions of its respective subset. Curve $1, k=0.2, R e=100$; curve $2, k=0.2, R e=150$; curve $3, k=0.3, R e=100$.

consider the case represented by curve 1 , for which $k=0.2$ and $R e=100$. For a fixed $A$, say 7.0, and a sufficiently small $B$, bubbles which do not escape to $y=\infty$ asymptotically accumulate at the stable equilibrium point inside the vortices. However, when $B$ is increased beyond the threshold value of 0.875 given by curve 1 , the buoyancy force becomes so large that no bubble can be held captive and all of them escape to $y=\infty$; this situation corresponds to the disappearance of the equilibrium points of the dynamical system of (1)-(2). When Re increases, so does the threshold values of $B$ for bubble entrapment, and curve 1 shifts upward (compare curves 1 and 2). Apparently, an increase in $R e$ results in a greater viscous drag so that it can counteract against a larger $B$. Since a larger $k$ corresponds to stronger Stuart vortices, an increase in $k$ results in the larger threshold values of $B$ for bubble entrapment; this is clearly illustrated by cases 1 and 3 of Figure 2 .

To gain further insight to the dynamics of bubbles in the Stuart vortex flow, let us assume that the viscous drag is the dominant force acting on the bubbles. Then, under the assumption of $\gamma=1 / A f_{\mathrm{d}} \ll 1$, we obtain from equation (1) the bubble velocity

$$
\mathbf{V} \sim \mathbf{u}+2\left[\mathbf{u} \cdot \nabla \mathbf{u}+B \mathbf{e}_{y}\right] \gamma+O\left(\gamma^{2}\right)
$$

which states that the velocity of a bubble is equal to that of the local fluid particle, except for a small perturbation of $O(\gamma)$. Taking the divergence of the "bubble flow field", equation (6), we obtain

$$
\nabla \cdot \mathbf{V}=4 \gamma \frac{k^{2}\left(\cos ^{2} x+\cosh ^{2} y\right)-\left(k^{2}+1\right) k \cos x \cosh y}{(\cosh y-k \cos x)^{4}}
$$




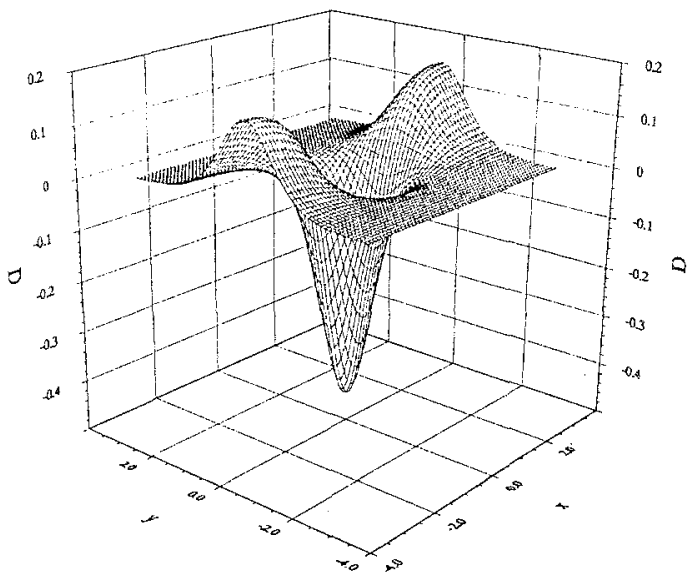

Fig. 3. Spatial variation of $D=(1 / 4 \gamma) \nabla \cdot \mathrm{V}$ for $k=0.25$.

In Figure 3, the quantity $D=(1 / 4 \gamma) \nabla \cdot \mathrm{V}$ is plotted for the case of $k=0.25$. Note that $D<0$ in the neighborhood of the vortex center, where it achieves its minimum value. Intuitively, this means that correct up to $O(\gamma)$, bubbles captured by a vortex all accumulate at the center of that vortex.

\section{Acknowledgements}

The research reported here is supported by the United Technologies Corporation through a gift and by the United States Office of Naval Research under Grant No. N00014-91-J-1252.

\section{References}

1. Auton, T.R., Hunt, J.C.R. and Prud'homme, M.: The force exerted on a body in inviscid unsteady non-uniform rotational flow. J. Fluid Mech. 197 (1988) 241-257.

2. Gañán-Calvo, A.M. and Lasheras, J.C.: The dynamics and mixing of small spherical particles in a plane, free shear layer. Phys. Fluids A 3 (1991) 1207-1217.

3. Maxey, M.R. and Riley, J.J.: Equation of motion for a small rigid sphere in a nonuniform flow. Phys. Fluids 26 (1983) 883-889.

4. Maxey, M.R.: The motion of small spherical particles in a cellular flow field. Phys. Fluids $\mathbf{3 0}$ (1987) 1915-1928.

5. Oliver, D.L.R. and Chung, J.N.: Flow about a fluid sphere at low to moderate Reynolds numbers. J. Fluid Mech. 177 (1987) 1-18.

6. Stuart, J.T.: On finite amplitude oscillations in laminar mixing layers. J. Fluid Mech. 29 (1967) $417-440$.

7. Thomas, N.H., Auton, T.R., Sene, K. and Hunt, J.C.R.: Entrapment and transport of bubbles by transient large eddies in multiphase turbulent shear flows. In: Physical Modelling of Multi-phase Flow. Cranfield (England): BHRA Fluid Engineering (1983) pp. 169-184. 
8. Thomas, N.H., Auton, T.R., Sene, K. and Hunt, J.C.R.: Entrapment and transport of bubbles by plunging water. In: Brutsaert, W. and Jirka, G.H. (eds.), Gas Transfer at Water Surfaces. Dordrecht: D. Reidel Publishing Company (1984) pp. 255-268.

9. Tio, K.-K., Liñán, A., Lasheras, J.C. and Gañan-Calyo, A.M.: On the dynamics of buoyant and heavy particles in a periodic Stuart vortex flow. J. Fluid Mech. (submitted). 